$\S=-1$

\title{
Access Channel Selection for WLAN using Fuzzy Expert System
}

\author{
Bakeel Maqhat, Mohd Dani Baba, Ruhani Ab Rahman and Anwar Saif \\ Centre for Computer Engineering Studies, Faculty of Electrical Engineering, Universiti Teknologi MARA, \\ 40450 Shah Alam, Selangor, Malaysia
}

\begin{abstract}
The tremendous increase in user demands for multimedia applications with its various quality of service (QoS) requirements has become essential for the operators to accommodate the demand for real-time services in IEEE 802.11 WLAN network. Scheduling mechanism is one of the challenging issues still open for research to fully support the various QoS requirements. In this paper, scheduling scheme is proposed to manage the channel access parameter between competitive nodes. An embedded fuzzy expert system is used to dynamically allocation these parameters to the competitive stations. The simulation results show that the proposed algorithm manages to optimize the overall system utilization.
\end{abstract}

Keywords: $x$ EDCA parameters, Frame Aggregation, IEEE 802.11n, fuzzy expert system

\section{Introduction}

The Enhanced Distributed Channel Access (EDCA) is introduced to guarantee differentiated and distributed access to the wireless medium accesses for nodes using eight various User Priorities (from 0 to 7). The User Priorities (UP) are named Traffic Categories (TCs) that are assigned to four Access Categories (ACs) (Wang, and W. Zhuang,2008). Every AC has its diverse of an EDCA function (EDCAF) and competes to access the channel using a set of EDCA parameters. The service differentiation between ACs is accomplished through the different period of time to sense the channel being idle (i.e., interframe spaces), the minimum contention window (CW_min), and the maximum contention window (CW_max), which required by the QSTA. The minimum idle period for sensing the channel is called the Arbitration Interframe Space (AIFS). The EDCF uses variant AIFSs for variant ACs to provide service differentiation, where The AIFS for a given AC is expressed as AIFS[AC]=AIFSN[AC] $\times$ SlotTime+SIFS. The value of AIFSN[AC] is an integer value equal or greater than one, and the highest priority of an AC will assign with the lowest value of an AIFSN[AC]. Furthermore, contention window lengths are specified for different ACs, in order to support high UP, allocating shorter CW to a higher priority AC (Y. Xiao et.al.,2005) In addition to the interframe space differentiation, distinct contention window lengths are specified for various ACs. Allocating shorter $\mathrm{CW}$ to the highest priority $\mathrm{AC}$ guarantee that the high priority is capable of sending data frames before the low priority AC. For AC_ $i,(i=0, . ., 3)$, the initial contention window size is CW_min [i] and the maximum contention window size is CW_max [i]. Similar to the backoff procedure in $802.11 \mathrm{DCF}$, in every failed transmission, the $\mathrm{CW}[\mathrm{i}]$ size is doubled till reach to maximum value CW_max [i]). The intended omission of scheduling in the IEEE $802.11 \mathrm{n}$ standard encourages the venders to innovate with their interpretation. The IEE802.11n scheduler is solely based on the priority technique of the legacy 802.11e Enhanced Distributed Channel Access (EDCA) scheduler. EDCA scheduler introduces four access categories (ACs); voice (AC_VO), video (AC_VI), background (AC_BK), and best effort (AC_BE). The unique queue of each AC prescribes specific waiting time for the channel access that relies on the ACs themselves. As a result, the higherpriority classes will be at an advantage in comparison to lowerpriority classes in the speed of channel access. A number of studies have been studied to overcome the limitations that affect traffics in WLAN. (M. Malli, et al) have introduced a scheme to enhance the QoS for ad hoc networks in IEEE802.11. The authors tray to improve the fundamental EDCF mechanism by applying an adaptive fast-backoff technique to enhance the overall throughput as well as a window-doubling technique at occupied intervals to conserve additional flows with most priority and enhance the fairness among those of the same priority particularly in the case of high network congestion. Authors in (Maqhat, et.al.2016) introduce the mechanism, which adjusts the transmission time based on the network conditions. The bandwidth allocation scheme between time and non time-based applications for WLAN networks is introduced in Maqhat, et.al.2016]. The fuzzy logic control is used in this algorithm to control and dynamically assign the required bandwidth to the various service classes according to their delay constraint and throughput. (S. Choi, et al.2008) have addressed the EDCF mechanism that supports QoS in IEEE802.11e MAC layer. According to simulation results, the EDCF can guarantee differentiated channel access between various priority traffic compared to legacy DCF.

Authors in (Baba, et al.,2014), introduced a real-time traffic scheduling algorithms based on an A-MSDU aggregation (RSA); A-MSDU aggregation after enabled retransmission (Saif, and Othman,2013) has become suitable and capable to meet the QoS constraints of time sensitive applications. RSA algorithm aims to transmission of different service classes in one superframe with taking into consideration the traffic characteristics; the packets from different access Categories are mapped to one queue called transmitting queue (TQ) rather than four queues as specified in the standard. In the TQ, the packets will be sorted in ascending order based on two criteria: expire time and priority. According to that, packets with the most urgent standing would be positioned ahead of others in the queue. During transmission, the scheduler takes the packets from the head of TQ and then channeled to the aggregated large frame that is named as the superframe. Due to the TQ 
contains packets from several AC in the RSA algorithm, thereby, the superframe will be aggregated from packets that belong to different classes. Therefore, the superframe priority will depend on its packets priorities. The RSA does not guarantee the idea of distinguishing between frames with various priorities during backoff process. Its access the channel like distributed coordination function that is assumed to supply a wireless medium access with the same probabilities to all contending nodes for the wireless medium access in a distributed method. Nevertheless, access the wireless medium with the same probabilities are unacceptable between nodes with various priority frames. In this paper, an Access Channel Selection for WLAN is proposed. Using an expert system to specified the channel access parameters; CW_min, CW max, and AIFS[AC] for each competitive node based on their traffic's priorities. Assigning smaller values of these parameters to a high-priority node can ensure that high-priority node obtains access to the channel faster than low-priority node.

For this purpose, an embedded fuzzy logic control is developed to dynamically manage the channel access parameter with low complexity. The fuzzy expert system requires two input variables namely, deadline of the ahead-of-line packet for time-based application and queue length for the non-time-based application. This system can dynamically assign channel access parameter between competitive nodes to access the channel effectively.

\section{Access Channel Selection for WLAN using Fuzzy Expert Sys- tem}

The Access Channel Selection for WLAN using Fuzzy Expert System (ACSF) manages the channel access parameters between competitive nodes. For this purpose, an embedded fuzzy logic control is developed to dynamically manage the channel access parameters with low complexity. The fuzzy expert system requires two input variables, the first input variable is Deadline of the ahead-of-line packet for real-time (DL) and the second input variable is the queue length ratio for the non-time-based application $\left(\mathrm{NRT}_{\mathrm{rql}}\right)$ that can be computed as shown in equation (1):

$$
\mathrm{NRT}_{\mathrm{rql}}=\mathrm{NRT}_{\mathrm{ql}} /\left(\mathrm{NRT}_{\mathrm{ql}}+\mathrm{RT}_{\mathrm{ql}}\right)
$$

Where $\mathrm{NRT}_{\mathrm{ql}}$ is non time-based queue length and $\mathrm{RT}_{\mathrm{ql}}$ is the time-based traffic queue length. This system can dynamically assign channel access parameters between competitive nodes to access the channel effectively

\section{Fuzzy Reasoning Inference Engine Control Model}

There are two variables and one single variable that are used by the embedded fuzzy logic control, the input, and the output respectively . This approach is very useful for wireless communications due to their non-linear environment which is hard to quantify the information precisely and develop a mathematical model. In this system, an innovative fuzzy logic control is designed for the linguistic information of which the vital design utilizes the expert information for the rule bases creation (Driankov et.al.,1996). Figure 1 illustrates the proposed fuzzy logic control. In this system, a separate based inference manner of Mamdani's design (Mamdani et.al,1977) were utilized, where the rules of the inference system are gathered into a single value. The fuzzy logic control contains three standard steps: fuzzification, fuzzy reasoning inference, and defuzzification. The aim is to actively analyse all incoming traffics and then crystallise them into a single fuzzy set. First of all, the fuzzification operation deals with the two input variables, DL and NRT_rql for the overall system. Then the reasoning inference mechanism, includes the rule-base, handle the input variables as given in Figure. 2. The result herein is a made-of decision, to represent the human expert process, which executes the linguistic behaviour to attain the calculated output value. At last, the defuzzification stage computes the crisp numerical values to attain the calculated access category number that guarantees the optimal access channel parameters for the station. Three linguistic levels have been determined for input variables DL of real-time packets namely (low, medium, high) and NRT_rql of non-real-time traffic. The understudy fuzzy logic control considers two variables as the inputs, three membership functions for each variable, as a result, the rule base exhibits nine rules thereafter (see Table I). The actively normalized scale for the DL input value is scaled between 0 and 200 millisecond (ms) and for the input variable NRT_rql formed from 0 to 1 while the output variable is one of the 3 values; 1,2 or 3 .

Output value (access category number (N_AC)) attained from the fuzzy inference system is the singleton value of one of the three values $(1,2,3)$, that is used to determine the channel access parameters for each station based on the corresponding service class. The access channel parameters selection is governed by the following equations:

$$
\begin{aligned}
& \text { MIN-CW }=\mathrm{CW}_{\min }\left[\mathrm{N}_{\mathrm{AC}}\right] \\
& \text { MAX-CW }=\mathrm{CW}_{\max }\left[\mathrm{N}_{\mathrm{AC}}\right] \\
& \text { AIFS }\left[\mathrm{N}_{\mathrm{AC}}\right]=\mathrm{SIFS}+\mathrm{AIFSN}\left[\mathrm{N}_{\mathrm{AC}}\right] \times \text { Slot Time }
\end{aligned}
$$

The parameter of RetryLimit (Number of retry attempts to send the packet) is subject to packet deadline for real-time traffic. The maximum retry attempts (RA_max) that recorded in the experiments will be the RetryLimit for non-real-time traffics. This algorithm able to increase utilization channel bandwidth by guarantee the maximum latency for traffic in the station to access the channel when the other stations are observed, without sacrificing their QoS requirements.

\section{Verification of the Fuzzy System}

The verification of the fuzzy logic control is performed by the following example to monitor the fuzzy inference process in the system, and to shows the values of the different inner variables. The example contains a set of if-statements with the activation degree of each logical rule and linguistic labels, for the given set of input values. Then shows the crisp value obtained by the inference process that is finally assigned to the output variable. Assume that, the two inputs variables, which specified by the scheduler as a 50 millisecond for the DL and a 0.92 for the NRT_rql. The fuzzy logic control considers the variables as system inputs, by evaluating the membership functions of Figure 2, which then could determine the linguistic values for the two input variables $[50,0.92]$ and could be shown as follow:

For instance, if the DL is 50 millisecond, which would be in the linguistic form, post fuzzification, rendered for low at grade membership of 0.6 and medium at grade membership of 0.4 (See Figure 2 (a)). And if NRT_rql is 0.92 , which would be, after fuzzification, in the medium grade membership of 0.25 and high grade membership of 0.75 (Figure 2 (b)). Following the fuzzy reasoning with association with rule based from Table I and Figure 2, could be read as such:

- If the DL is low (0.60) AND $\mathrm{NRT}_{\text {rql }}$ is medium (0.25) THEN the N_AC value is 3 which means VoIP access category (AC_VoIP) at grade membership of 0.20 [Rule 1].

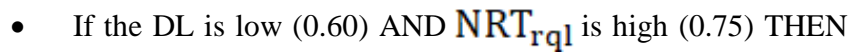
the N_AC value is 3 (AC_VoIP) at grade membership of 0.56 [Rule 2].

- If the DL is medium (0.40) AND NRT $_{\mathrm{rql}}$ is medium (0.25) AND THEN the N_AC value is 2 (AC_Video ) at grade membership of 0.2 [Rule 4].

- If the DL is medium (0.40) AND NRT rql $_{\text {is high (0.75) }}$ THEN the N_AC value is 1 (AC_NRT) at grade membership of 0.28 [Rule 5]. 
According to, foregoing rules and applying Mamdami's inference we can infer that the output value will be three (AC_VoIP) at grade of membership 0.20 and 0.56 , two (AC_Video) at grade membership of 0.20 and one (AC_NRT) at grade membership of 0.28 . After defuzzification we compute the final value of output N_AC which produces a singleton value of 3 for AC_VoIP. It will be used as a number of access category to select an optimal channel access parameters with taking into consideration QoS requirements. For every two input variables; DL and $\mathrm{NRT}_{\mathrm{rq}}$, the fuzzy expert system produces one crisp value N_AC. Further examples can be found in Table II.

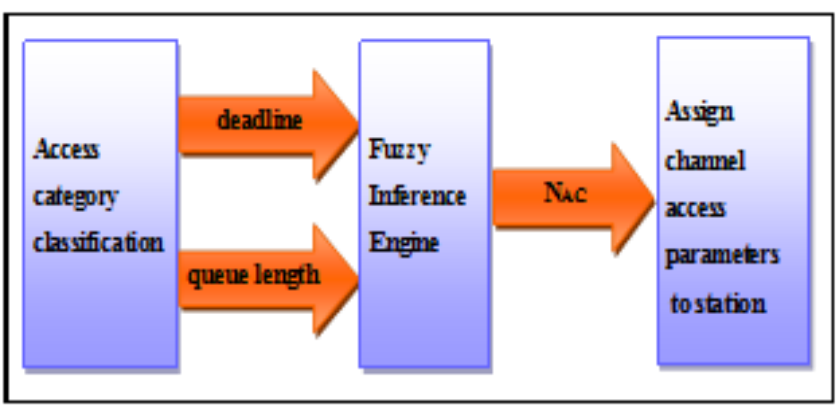

Figure 1. Model of Fuzzy logic for ACSF

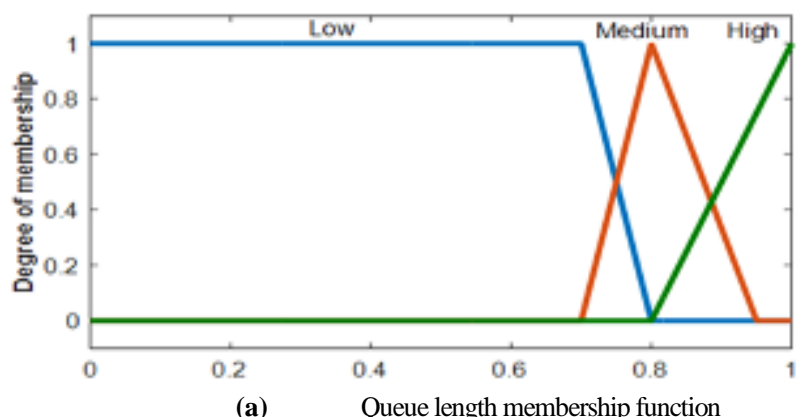

(b)

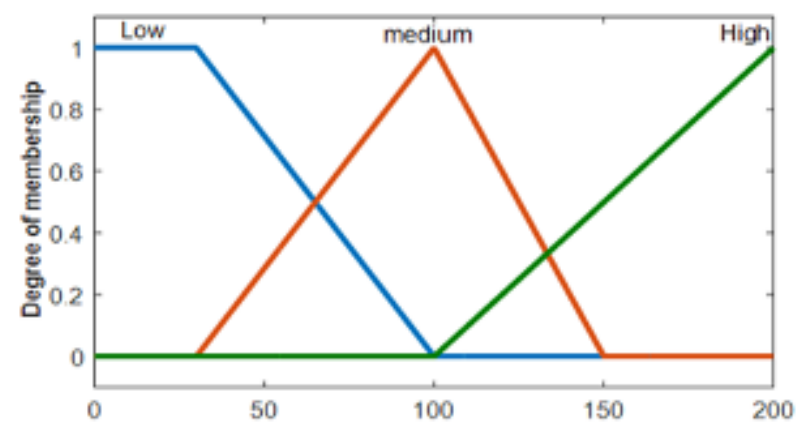

(a) Packet Deadline membership function

(b)

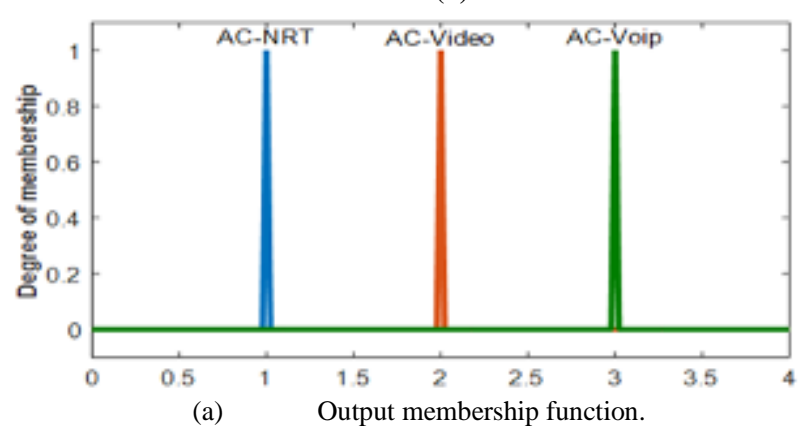

Figure. 2. Membership functions for inputs and output.

Table 1. Fuzzy rule bases

\begin{tabular}{|l|l|l|l|}
\hline Rules No & DL & Queue leng. & Output $\mathbb{N}_{\text {AC }}$ \\
\hline 0 & Low & low & 3 \\
1 & Low & Medium & 3 \\
2 & Low & High & 3 \\
3 & Medium & Low & 2 \\
\hline
\end{tabular}

\begin{tabular}{|l|l|l|l|}
\hline 4 & Medium & Medium & 2 \\
5 & Medium & High & 1 \\
6 & High & Low & 2 \\
7 & High & Medium & 2 \\
8 & High & High & 1 \\
\hline
\end{tabular}

Table 2. Examples of input variables and their crisp values

\begin{tabular}{|c|c|c|}
\hline \multicolumn{2}{|c|}{ Input } & Output $\left(\mathrm{N}_{\mathrm{AC}}\right)$ \\
\hline $\mathrm{DL}$ & $\mathrm{NRT}_{\mathrm{rq} \mathrm{l}}$ & \\
\hline 50 & 0.92 & 3 \\
60 & 0.30 & 3 \\
& 0.95 & 2 \\
\hline 160 & 0.75 & 2 \\
\hline 190 & 0.80 & 2 \\
\hline
\end{tabular}

\section{Results and Discussions}

The network simulator (NS2) was utilised for several simulation experiments to evaluate different traffics scheduling especially in overall system throughput, packet loss ratio, and average delay. The scheduling scheme deals with multimedia traffic. Moreover, we have used the simulation point-to-point usage model. This scenario contains a single-hop WLAN, to avoid hidden nodes in the wireless system the transmission power is high for the whole high throughput nodes. Each node operates over a $20 \mathrm{MHz}$. Moreover, the algorithm deals with the multimedia traffics; VoIP (120 bytes) and Video conferencing (512 bytes) for real-time class services. And the Best efforts (500 bytes) with traffic rate $1.07 \mathrm{Mbps}$, and Background (512 bytes) for non-real-time class services. The EDCF parameters for the traffic are listed in Table III. The physical rate are set to $150 \mathrm{Mbps}$ and $300 \mathrm{Mbps}$ and basicrate is set to $54 \mathrm{Mbps}$, the number of stations increased from 10 to 70 for every 10 units.

Figures 3-5, show the packet loss ratio (PLR) of ACSF scheme comparison to RSA algorithm. As a result of fewer contending network nodes, the PLR will therefore be small because of the fewer number of superframes competing to send at the network. However, the PLR will grow with the corresponding increase in stations' numbers. Furthermore, the excellent performance of the proposed scheme can be seen in the Figures through the reduction of PLR for high bit error rate and high network congestion. This is because the scheme allows the highest priority station to occupy the medium channel prior the stations that have low priority. There is a significant enhancement in VoIP packet loss, as shown in Figure 3, of about 22\% under the two data rates, whereas figure. 4 displays the video conferencing enhancement that is about $33 \%$ and $43 \%$ under $150 \mathrm{Mbps}$ and $300 \mathrm{Mbps}$, respectively. The non-real-time traffic (BE and BK) score an enhancement of about $38 \%$ under data rate of $150 \mathrm{Mbps}$ and $50 \%$ under $300 \mathrm{Mbps}$ data rate, see figure 5 . The high performance of packet loss ratio is attributed to the AFR scheme can dynamically distinguish the data of each contending station and determining the lowest deadline traffic and gives them the higher chance to access the channel and to be transmitted.

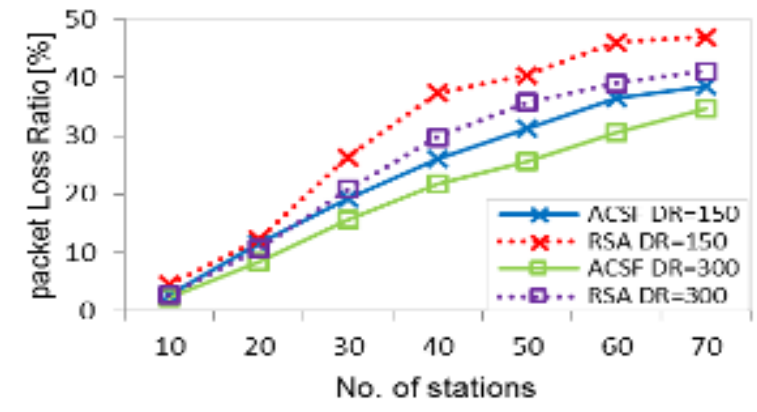

Figure. 3. Packet loss ratio for VoIP 


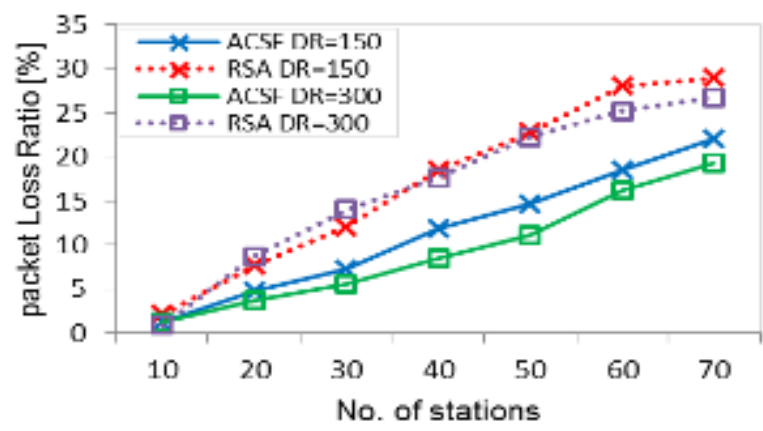

Figure.4. Packet loss ratio for Video Conf.

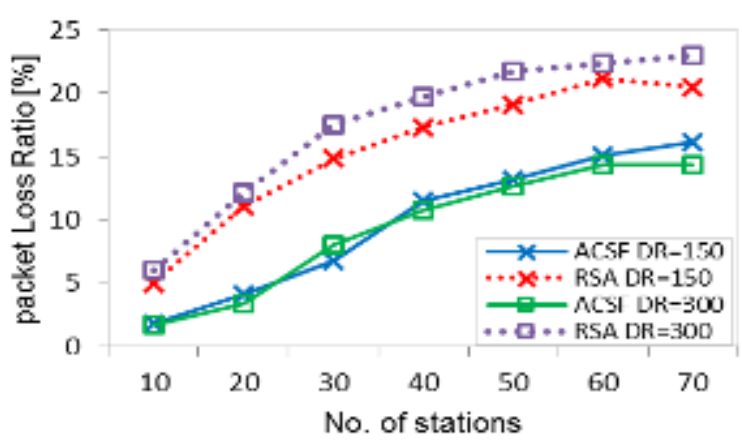

Figure.5. NRT packet loss ratio

The lesser the contending nodes the lesser average delay time is found because the nodes will have repeatedly access to the wireless channel. Likewise, the packets will not face longer queue waiting time. On the other hand, growing number of stations will result in the rise of the average delay. That is primarily due to both, the increase in the contention time and supreframe re-transmission time. The existence of large number of stations in the wireless environment can lead to two or more nodes to sense that the channel is idle at the same time. If these stations send their backlogged packets, then the collision will occur and the transmitted packets will be lost. The stations will try to retransmit their packets after a random period of time. Thus, the collision probability will experience an increase due to the potential increase of nodes. As the retransmission attempts increase the service rate will not commensurate with the arrival rate, therefore the packets will experience a long queuing delay, which in turns affects the system delay.

The average delay of the ACSF and RSA schemes is shown in figures 6 and 7. ACSF algorithm achieves smallest average delay compare to RSA due to ACSF ability to serve the highest priority station first by means of assign the smaller values of channel access parameters to a high-priority node to guarantee the station with maximum latency traffic to access the channel and transmit its packets at an adequate time before their expiration time. While RSA gives equal probabilities to all competition nodes to access the wireless medium. Thus, in ACSF algorithm, the frames will not face longer delay in the queue. The significant enhancement for the ACNF scheme compares to the RSA scheme is about $15 \%$ on average for VoIP under different data rates and about 18 and $21 \%$ under $150 \mathrm{Mbps}$ and $300 \mathrm{Mbps}$ respectively, for video conferencing.

Figure 8 describe the throughput performance of the proposed ACSF algorithm experiencing with varied number of nodes. However, for extended number of nodes, the packet collision is inevitably frequent and affects the system performance considerably. The overall system throughput persists reducing while the number of nodes grows. Nonetheless, the overall system throughput of the ACSF algorithm approaches about $38 \mathrm{Mbps}$ and 53Mbps whilst, the RSA algorithm hardly reaches $33 \mathrm{Mbps}$ and $49 \mathrm{Mbps}$ at high traffic load150Mbps and 300Mbps respectively.

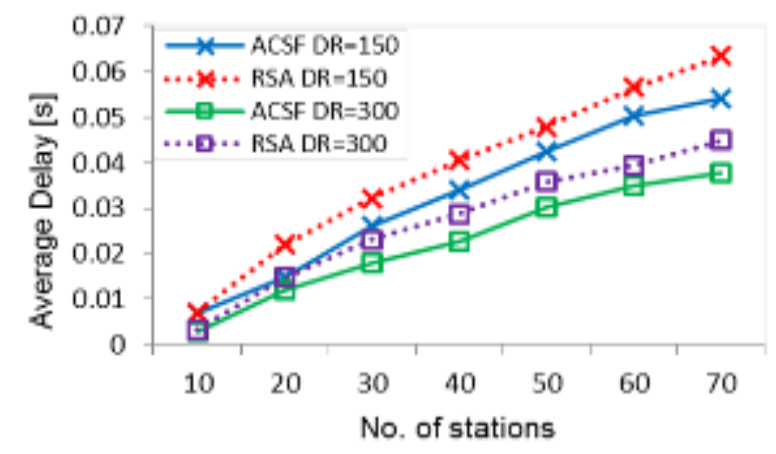

Figure. 6. VoIP average delay.

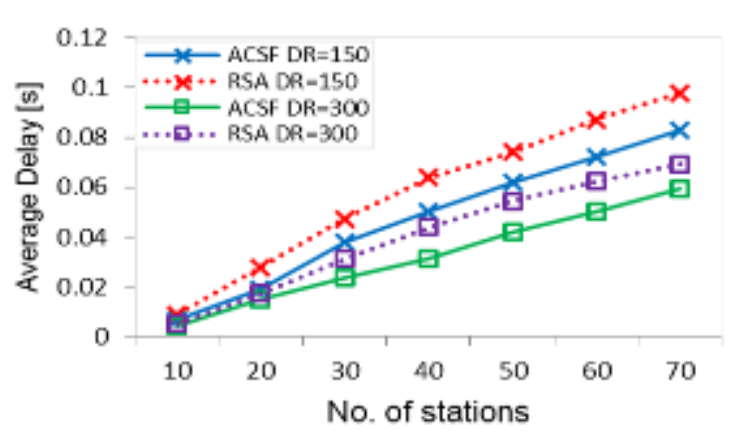

Figure.7. Video Conf. average delay.

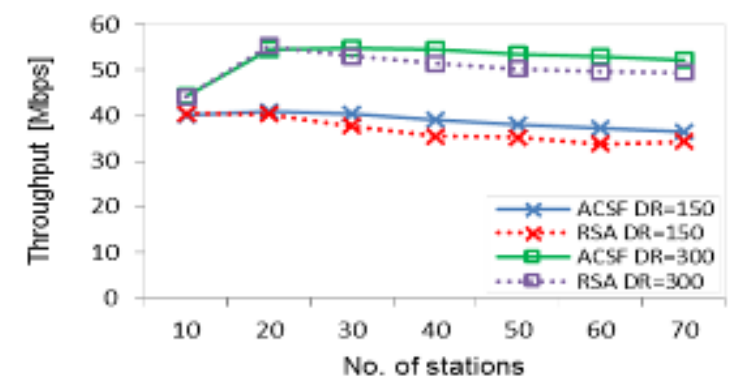

Figure. 8. System throughput.

Table 3. EDCF parameters for the traffic

\begin{tabular}{|l|l|l|l|}
\hline & VoIP & Video & NRT \\
\hline Priority & 3 & 2 & 1 \\
$\mathrm{CW}_{\min }$ & 7 & 15 & 31 \\
$\mathrm{CW}_{\max }$ & 15 & 31 & 1023 \\
AIFSN & 1 & 1 & 3 \\
Delay bound & $30 \mathrm{~ms}$ & $100 \mathrm{~ms}$ & - \\
\hline
\end{tabular}

\section{Conclusion}

In this paper, Access Channel Selection for WLAN using Fuzzy Expert System (ACSF) has been described. ACSF dynamically manages the selection process for the channel access parameters. It used the maximum latency of ahead-of-line packet for timebased application and queue length for the non-time-based application to manipulate requirements of the diverse traffic type. ACSF can dynamically distinguish the data of each station contending; and it adaptively allocates channel access parameters for all stations contending, considering their traffics deadline, and improves access for non-real time stations. The results indicate that ACSF attains superior performance over that of the RSA algorithm. Since the RSA algorithm did not take into account the traffic priorities for each station during the channel access process

\section{References}

[1] Wang, P. 2008 "A token-based scheduling scheme for WLANs supporting voice/data traffic and its performance analysis," 
Wireless Communications, IEEE Transactions on, vol. 7, no. 5, pp. 1708-1718.

[2] Xiao,Y , 2005 "Performance analysis of priority schemes for IEEE 802.11 and IEEE 802.11 e wireless LANs," Wireless Communications, IEEE Transactions on, vol. 4, no. 4, pp. 1506-1515.

[3] Malli, M, Q."Adaptive fair channel allocation for QoS enhancement in IEEE 802.11 wireless LANs." pp. 3470-3475.

[4] Maqhat, B, 2016. Adaptive Packet Scheduling Scheme Based on Network Conditions in WLAN Using Fuzzy System. In Theory and Applications of Applied Electromagnetics. Springer.

[5] Maqhat, B. 2016"Adaptive Bandwidth Allocation and Latency Guarantee for WLAN Networks using Fuzzy Logic Control". International Journal of Control Theory and Application, I JCTA, 9(9), 2016, pp. 3015-3027.

[6] Choi, S. 2003, "IEEE802.11e contention-based channel access (EDCF) performance evaluation.",. ICC'03. IEEEInternational Conference on Communications pp. 1151-1156.

[7] Baba, M. 2014, "Scheduler algorithm for IEEE802. 11n wireless LANs," International Journal of Future Computer and Communication, vol. 3, no. 4, pp. 222, 2014

[8] Saif, A. "SRA-MSDU: Enhanced A-MSDU frame aggregation with selective retransmission in $802.11 \mathrm{n}$ wireless networks," Journal of Network and Computer Applications, vol. 36, no. 4, pp. 1219-1229, 2013.

[9] Driankov, D. 2013 An introduction to fuzzy control: Springer Science \& Business Media.

[10] King, P, 1977 "The application of fuzzy control systems to industrial processes," Automatica, vol. 13, no. 3, pp. 235-242, 1977. 